Systematic Review

\title{
e Transforaminal Versus Interlaminar Approaches to Epidural Steroid Injections: A Systematic Review of Comparative Studies for Lumbosacral Radicular Pain
}

George C. Chang Chien, DO1, Nebojsa Nick Knezevic, MD, PhD², Zack McCormick, MD, Samuel K. Chu, MD ${ }^{1}$, Andrea M. Trescot, MD², and Kenneth D. Candido, MD²

From: ${ }^{1}$ Department of Physical Medicine and Rehabilitation, Rehabilitation Institute of Chicago, Northwestern McGaw Medical Center, Chicago, IL; ${ }^{2}$ Department of Anesthesiology, Advocate Illinois Masonic Medical Center, Chicago, IL; Department of Anesthesiology, University of Illinois, Chicago, IL; ${ }^{3 P a i n}$ and Headache Center, Eagle River, AK

Dr. Chien, Dr. McCormick and Dr. Chu are Residents with the Department of Physical Medicine and Rehabilitation, Rehabilitation Institute of Chicago, Northwestern McGaw Medical Center, Chicago, IL. Dr. Knezevic is Director of Anesthesiology Research and Clinical Assistant Professor, Department of Anesthesiology, Advocate Illinois Masonic Medical Center, Chicago, IL; and the Department of Anesthesiology,

University of Illinois, Chicago, IL. Dr.

Trescot is Medical Director of the Pain and Headache Center, Eagle River, AK. Dr. Candido is Chairman and Professor with the Department of Anesthesiology, Advocate Illinois Masonic Medical Center, Chicago, IL; and the Department of Anesthesiology, University of Illinois, Chicago.

Address Correspondence: Kenneth D. Candido, M.D. Department of Anesthesiology, Advocate Illinois Masonic Medical Center, Chicago, IL 836 W. Wellington Ave. Suite 4815 Chicago, IL 60657 Email: kdcandido@yahoo.com

Disclaimer: There was no external funding in the preparation of this manuscript.

Manuscript received: 10-28-2013 Revised manuscript received: o1-21-2014 Accepted for publication: 04-08-2014

Free full manuscript: www.painphysicianjournal.com
Background: The superiority of transforaminal epidural steroid injections (TFESI) vs. interlaminar epidural steroid injections (ILESI) for treating unilateral lumbosacral radicular pain (LSRP) is unproven.

Objective: To assess studies comparing TFESI to ILESI for unilateral LSRP for pain relief and functional improvement.

Study Design: Systematic review of comparative studies.

Methods: A systematic literature search was conducted using the Cochrane Central Register of Controlled Trials, PubMed, and Scopus databases for trials reported in English. Studies meeting the Cochrane Review criteria for randomized trials and the AHCQ criteria for observational studies were included. Evidence was graded using the USPSTF classification.

Results: Five (prospective) and 3 (retrospective) studies were included assessing 506 patients. Statistical analysis was calculated only utilizing the 5 prospective studies and consisted of 249 patients with an average of 3.2 months follow-up. In the short-term (2 weeks), there was a $15 \%$ difference favoring TFESI vs. ILESI for pain relief. There was no efficacy difference at one or 6 months. Combined pain improvements in all 5 prospective studies revealed $<20 \%$ difference between TFESI and ILESI (54.1\% vs. $42.7 \%$ ). There was slightly better functional improvement in ILESI groups (56.4\%) vs. TFESI groups $(49.4 \%)$ at 2 weeks. Combined data showed slight differences (TFESI $40.1 \%$ and ILESI $44.8 \%$ ).

Limitations: The limitations of this systematic review include the relative paucity of comparative studies.

Conclusions: The findings show that both TFESI and ILESI are effective in reducing pain and improving functional scores in unilateral LSRP. In the treatment of pain, TFESI demonstrated non-clinically significant superiority to ILESI only at the 2-week follow-up. Based on 2 studies, ILESI demonstrated non-clinically significant superiority to TFESI in functional improvement.

Key words: Radiculopathy, epidural steroids, transforaminal, interlaminar, systematic review

Pain Physician 2014; 17:E509-E524 
T ransforaminal epidural steroid injections (TFESI) and interlaminar epidural steroid injections (ILESI) are commonly performed procedures for the management of unilateral lumbosacral radicular pain (LSRP). However controversy exists about the superior efficacy of one of these 2 interventional approaches respective to the other. Unilateral LSRP is thought to originate from inflammation in the proximity of a damaged intervertebral disc or a narrowed neural foramen that irritates an exiting spinal nerve root (14). Thus corticosteroids are commonly used to reduce inflammation in the epidural space (5-9). The purported advantage of TFESI over ILESI is attributed to enhanced deposition of medication in closest proximity to the pain generators found in the ventral epidural space (10), and hence reaching the targeted pain generators with a smaller dose of medication. Some evidence suggests that TFESI allow for greater ventral epidural spread of corticosteroid (11), and ventral epidural spread of corticosteroid has been associated with superior pain and functional outcome improvements (11). Data from multiple studies and systematic reviews of the published data support the utility of TFESI, and have shown that lumbar TFESI are effective for reducing pain, improving functionality, preventing spine surgery, and for treating radiculopathic pain (1215). As a reflection of this perceived enhanced efficacy, there has been an exponential growth in the utilization of TFESI according to the Centers for Medicare and Medicaid Services (CMS) studies on utilization (15-17). Analysis of CMS data demonstrated that during the period from 2000 to 2011, utilization of lumbosacral TFESI grew at an annual rate of $20.4 \%$, whereas lumbosacral interlaminar and caudal injections grew at a comparatively modest annual rate of $2 \%(16)$.

Despite the touted advantages for TFESI, the technique has been noted to carry certain unique risks. TFESI are more often implicated in severe, permanent complications compared to ILESI, including intravascular injection in up to $23 \%$ of lumbar epidural injection cases (18), which can lead to spinal cord infarction and paralysis (19-21). Intravascular injection with TFESI can occur even with the use of digital subtraction angiography or following a negative lidocaine anesthetic test dose (19). TFESI, compared to ILESI, are associated with a 12fold increased risk of intradiscal injection $(22,23)$, which can potentially weaken the disc or lead to discitis (24). Additionally, TFESI do not decrease the risk of known complications of ILESI, such as dural and subdural punctures (25), hematoma formation (26-30), and cauda equina syndrome (31). With regards to global outcome in an individual with lumbosacral radicular pain, the increased risk of complications associated with TFESI must be weighed against possibility for superior pain relief and functional outcomes that reduce the rate of spinal surgery (32), which is itself associated with significant vascular, neurologic, urologic, and infectious complications (33-35). However it remains unclear if TFESI result in clinically or statistically significant improvement in pain and functional outcomes compared to ILESI.

The existing data suggests long-term efficacy benefits are greater for TFESI compared to ILESI (12$14,32,36)$. However, conflicting data also exist for equivalent benefits between the 2 techniques as well (37-39). Many authors have performed systematic reviews on the efficacy of epidural steroid injections leading to a range of conclusions. However, there are only few well-designed, prospective, randomized, double-blind, controlled studies. Importantly, several studies have directly compared these 2 approaches with conflicting results. Our goal was to systematically review, grade the evidence, and perform a meta-analysis of the existing head-to-head comparative studies. In this review, we identified the available published data comparing the short- and long-term efficacy of TFESI and ILESI for improving pain and functionality in individuals with unilateral lumbosacral radicular pain.

\section{Methods}

\section{Study Design}

The standards set by the Preferred Reporting Items for Systematic Reviews and Meta-Analyses (PRISMA) guidelines were used to construct this systematic review. The 27-item checklist and 4-phase flow diagram were accessed from the PRISMA website on July 29, 2013.

\section{Eligibility Criteria}

For inclusion in the present systematic review, papers had to report the results of clinical studies evaluating transforaminal versus interlaminar epidural steroid injections. More specifically, articles had to meet the following eligibility criteria: (1) human adult men and women ( $>18$ years) suffering from unilateral lumbosacral radicular pain were evaluated; (2) patients' symptoms were secondary to intervertebral disc herniations/degeneration; (3) patients were followed-up a minimum of 2 weeks; (4) papers were published in English prior to August 2013. 
Randomized controlled trials were identified as the primary studies for analysis. To be included, for statistical analysis, patients must have been randomized to TFESI or ILESI.

Non-randomized studies were also identified for secondary review if a small number of RCTs were found.

Studies were excluded from analysis if they had poorly described needle placement methodology, did not use fluoroscopic guidance for needle placement, did not report standardized pain scores at defined follow-up intervals, or did not provide statistical analyses of their results. Review articles, letters to the editor, and studies that did not directly compare TFESI versus ILESI as epidural steroid injections were excluded from consideration.

\section{Literature Search}

We conducted a comprehensive literature search of Medline (PubMed)®, Cochrane Central Register of Controlled Trials (CENTRAL), and Scopus databases for relevant English language publications from 1966 through August 2013 in order to identify studies that specifically compared lumbar transforaminal to interlaminar epidural steroid injections in the treatment of unilateral lumbosacral radicular pain. Search terms included, "Transforaminal Epidural Steroid Injection"; "Interlaminar Epidural Steroid Injection"; "Efficacy of Transforaminal Epidural Steroid Injection"; "Efficacy of Interlaminar Epidural Steroid Injection"; "Transforaminal versus Interlaminar Epidural Steroid Injection"; "Efficacy of Transforaminal versus Interlaminar Epidural Steroid Injection"; "Selective Nerve Root Block versus Interlaminar Epidural Steroid Injection"; "Transforaminal versus Interspinous Injections"; and "Nerve Root Block versus Interspinous Injection."

References from each article directly comparing the 2 approaches, in addition to review articles discussing efficacy of the 2 approaches, were cross-referenced in order to identify additional relevant studies. The literature search methodology was developed by the first 3 authors (GCC, ZM, SK), and conducted by 2 independent reviewers (GCC and SK). Any differences in selected papers for inclusion and exclusion were resolved by consensus.

\section{Outcome Parameters}

The primary outcome measure was efficacy determined as "degree of pain relief" (visual or verbal analog pain score; numeric pain rating scale). The secondary outcome measure was functional improvement
(Oswestry Disability Index, Depression Numeric Rating Scale, city block walking tolerance, Global Perceived Effect, and Oswestry Low Back pain scale-EIFEL). We considered a difference in pain scores of at least $30 \%$ as being clinically significant. In order to determine the duration of effect measured, we looked for follow-ups at regular intervals, including at 2 weeks, 4 weeks, and 6 months with 4 weeks being considered a short-term effect; and 6 months being considered an intermediate-term effect. We accepted this duration upon recommendations suggested in the above-referenced Cochrane review (40).

The Cochrane Review criteria (41) for randomized trials and the Agency for Health Care Quality (AHCQ) criteria (42) for observational studies were applied to ensure that the studies considered used proper methodology. Studies that scored higher than 50 out of 100 using these measures were included (Tables 1 and 2). Each study was scored independently by 2 of the authors (ZM, SKC), and a third author (GCC) independently reviewed studies that were scored differently by the first 2 authors. This weighted scoring system has been used in multiple systematic reviews of interventional treatment for back pain $(12,14,41,43-58)$.

\section{Risk of Bias}

The Cochrane Risk of Bias Tool was utilized to systematically assess for bias in identified prospective studies. The support judgments for each manuscript can be found in the (Table 3). The Newcastle-Ottawa Scale (NOS) was used to assess the quality of the identified non-randomized trials (wells). The lead (GCC) and secondary authors (NNK) assessed each study for bias to ascertain a consensus grading (Table 4).

\section{Grading Quality of Evidence}

The United States Preventive Services Task Force (USPSTF) level of evidence classification (59) (Table 5) was used to grade the level of evidence reported in the literature describing the comparison of TFESI vs. ILESI for the treatment of lumbosacral radicular pain.

\section{Statistical Analysis}

Statistical analysis was performed using SPSS (IBM SPSS Statistics 20, Chicago, IL) and MedCalc 12.7.0 (Ostend, Belgium) software. Differences in age, pain, and functional improvement between TFESI and ILESI were analyzed using independent samples t-test. Differences in gender, level of injection, or type of corticosteroids between the 2 groups were analyzed by $\chi^{2}$ test. For- 
Table 1. Methodological assessment of randomized controlled trials comparing the efficacy of transforaminal to interlaminar epidural steroid injections.

\begin{tabular}{|c|c|c|c|c|c|c|}
\hline & $\begin{array}{c}\text { Weight } \\
\text { (points) }\end{array}$ & $\begin{array}{c}\text { Gharibo et al } \\
\qquad(68)\end{array}$ & $\begin{array}{l}\text { Candido et } \\
\text { al (37) }\end{array}$ & $\begin{array}{l}\text { Rados et } \\
\text { al (67) }\end{array}$ & $\begin{array}{c}\text { Ackerman } \\
\text { and Ahmad } \\
\text { (11) }\end{array}$ & $\begin{array}{c}\text { Kolsi et } \\
\text { al (69) }\end{array}$ \\
\hline Study Population & 35 & & & & & \\
\hline Homogeneity & 2 & 2 & 2 & 2 & 2 & 2 \\
\hline Comparability of relevant baseline characteristics & 5 & 3 & 3 & 3 & 4 & 5 \\
\hline Randomization procedure adequate & 4 & 4 & 4 & 4 & 4 & 2 \\
\hline $\begin{array}{l}\text { Drop-outs described for each study group } \\
\text { separately }\end{array}$ & 3 & 3 & 3 & 3 & 3 & 3 \\
\hline$\leq 20 \%$ loss for follow-up & 2 & 2 & 2 & 2 & 2 & 2 \\
\hline$\leq 10 \%$ loss for follow-up & 2 & 2 & 2 & 2 & 2 & 2 \\
\hline$>50$ subject in the smallest group & 8 & 0 & 0 & 0 & 0 & 0 \\
\hline$>100$ subjects in the smallest group & 9 & 0 & 0 & 0 & 0 & 0 \\
\hline Interventions & 25 & & & & & \\
\hline Interventions included in protocol and described & 10 & 10 & 10 & 10 & 10 & 10 \\
\hline Pragmatic study & 5 & 5 & 5 & 5 & 5 & 5 \\
\hline Co-interventions avoided or similar & 5 & 5 & 5 & 5 & 5 & 5 \\
\hline Placebo-controlled & 5 & 0 & 0 & 0 & 0 & 0 \\
\hline Effect & 30 & & & & & \\
\hline Patients blinded & 5 & 5 & 5 & 5 & 5 & 5 \\
\hline Outcome measures relevant & 10 & 6 & 2 & 6 & 6 & 6 \\
\hline Blinded outcome assessments & 10 & 10 & 0 & 0 & 10 & 10 \\
\hline Follow-up period adequate & 5 & 3 & 5 & 5 & 5 & 3 \\
\hline Data Presentation and Analysis & 10 & & & & & \\
\hline Intention-to-treat analysis & 5 & 0 & 0 & 0 & 5 & 0 \\
\hline $\begin{array}{l}\text { Frequencies of most important outcomes } \\
\text { presented for each treatment group }\end{array}$ & 5 & 5 & 5 & 5 & 5 & 5 \\
\hline Total Score: & 100 & 65 & 53 & 57 & 63 & 65 \\
\hline
\end{tabular}

* Above Cochrane Review criteria for randomized trials adapted from ref. 41

est plots and $\mathrm{I}^{2}$ calculation were performed using the MedCalc 12.7.0 software.

\section{Results}

\section{Literature Search}

In our exhaustive literature search, 1,007 references were identified using the key words. Subtracting 625 duplicate items, 372 articles were screened for review. We identified 12 studies that specifically compared transforaminal to interlaminar approaches of epidural steroid injection for the treatment of unilateral LSRP which were subsequently assessed for eligibility (Fig. 1).

Kraemer et al (60) compared non-conventional interlaminar epidural steroid injections with epidural perineural injections, wherein a needle was passed towards the medial neuroforamen from the interlaminar space. This study was excluded from analysis due to a lack of consistent use of fluoroscopic guidance, and the unique procedural approach employed. The largest study comparing TFESI to ILESI was from Lee et al (38), but it was excluded from analysis as patients with lumbosacral radicular symptoms were paradoxically excluded from their study. The use of fluoroscopy is acknowledged as being superior to blind epidural steroid injection due to the high false positive rate with the loss of resistance (LOR) technique without fluoroscopic 
Table 2. Methodological Assessment of retrospective studies comparing the efficacy of transforaminal to interlaminar epidural steroid injections.

\begin{tabular}{|c|c|c|c|}
\hline Criterion & $\begin{array}{c}\text { Smith et al } \\
(72)\end{array}$ & $\begin{array}{c}\text { Lee et al } \\
\quad(73)\end{array}$ & $\begin{array}{l}\text { Schaufele } \\
\text { et al (70) }\end{array}$ \\
\hline \multicolumn{4}{|l|}{ 1. Study Question } \\
\hline Clearly focused and appropriate question & 2 & 2 & 2 \\
\hline \multicolumn{4}{|l|}{ 2. Study Population } \\
\hline Description of study population & 2 & 2 & 0 \\
\hline Sample size justification & 0 & 0 & 0 \\
\hline \multicolumn{4}{|l|}{ 3. Comparability of Subjects } \\
\hline Specific Inclusion/Exclusion Criteria for all groups & 5 & 5 & 5 \\
\hline Criteria applied equally to all groups & 3 & 3 & 3 \\
\hline Comparability of groups at baseline with regard to disease status and prognostic factors & 3 & 2 & 2 \\
\hline Study Groups comparable to non-participants with regard to confounding factors & 3 & 3 & 0 \\
\hline Use of concurrent controls & 0 & 0 & 0 \\
\hline Comparability of follow-up of each group at assessment & 3 & 2 & 3 \\
\hline \multicolumn{4}{|l|}{ 4. Exposure or Intervention } \\
\hline Clear definition of exposure & 5 & 5 & 5 \\
\hline Measurement method standard, valid and reliable & 3 & 3 & 3 \\
\hline Exposure measured equally in all study groups & 3 & 3 & 3 \\
\hline \multicolumn{4}{|l|}{ 5. Outcome Measures } \\
\hline Primary/secondary outcomes clearly defined & 5 & 5 & 5 \\
\hline Outcomes assessed blind to exposure or intervention & 5 & 3 & 0 \\
\hline Method of outcome assessment standard, valid and reliable & 5 & 5 & 5 \\
\hline Length of follow-up adequate for question & 5 & 5 & 0 \\
\hline \multicolumn{4}{|l|}{ 6. Statistical Analysis } \\
\hline Statistical tests appropriate & 5 & 5 & 5 \\
\hline Multiple comparisons taken into consideration & 1 & 2 & 3 \\
\hline Modeling and multivariate techniques appropriate & 2 & 2 & 2 \\
\hline Power calculation provided & 0 & 0 & 0 \\
\hline Assessment of confounding & 0 & 0 & 0 \\
\hline Dose-response assessment if appropriate & 2 & 2 & 0 \\
\hline \multicolumn{4}{|l|}{ 7. Results } \\
\hline Measure of effect for outcomes and appropriate measure of precision & 3 & 3 & 5 \\
\hline Adequacy of follow-up for each study group & 3 & 3 & 3 \\
\hline \multicolumn{4}{|l|}{ 8. Discussion } \\
\hline Conclusions supported by results with possible biases and limitations taken into consideration & 4 & 5 & 5 \\
\hline \multicolumn{4}{|l|}{ 9. Funding or Sponsorship } \\
\hline Type and sources of support for study & 5 & 3 & 5 \\
\hline TOTAL SCORE & 78 & 73 & 64 \\
\hline
\end{tabular}

${ }^{\star}$ Above Agency for Health Care Quality criteria for observational studies adapted from West $S$ et al (42). 
Table 3. Risk for bias in prospective studies.

\begin{tabular}{|c|c|c|c|c|c|c|c|}
\hline & Sequence generation & $\begin{array}{l}\text { Allocation } \\
\text { concealment }\end{array}$ & Blinding & $\begin{array}{l}\text { Incomplete } \\
\text { outcomes } \\
\text { Data }\end{array}$ & $\begin{array}{l}\text { Selective } \\
\text { outcomes } \\
\text { reporting }\end{array}$ & $\begin{array}{l}\text { Other } \\
\text { sources of } \\
\text { bias }\end{array}$ & Confounding \\
\hline $\begin{array}{l}\text { Gharibo } \\
\text { et al (68) }\end{array}$ & $\begin{array}{l}\text { Low Risk } \\
\text { "Participants were } \\
\text { randomly assigned to } \\
\text { one of } 2 \text { groups using } \\
\text { a computer-generated } \\
\text { randomization } \\
\text { table." }\end{array}$ & $\begin{array}{l}\text { Low risk } \\
\text { "Allocation to } \\
\text { injection } \\
\text { type was randomly } \\
\text { computer } \\
\text { generated." }\end{array}$ & $\begin{array}{l}\text { Low risk } \\
\text { The } \\
\text { interventionalist } \\
\text { was blind to } \\
\text { participant data. }\end{array}$ & Low risk & Low risk & $\begin{array}{l}\text { Low risk } \\
\text { No } \\
\text { conflicts } \\
\text { reported. }\end{array}$ & Low risk \\
\hline $\begin{array}{l}\text { Candido } \\
\text { et al (37) }\end{array}$ & $\begin{array}{l}\text { Low Risk } \\
\text { "Patients } \\
\text { were randomly } \\
\text { assigned to one of two } \\
\text { groups using a } \\
\text { computer-generated } \\
\text { randomization table." }\end{array}$ & Unclear risk & Unclear risk & $\begin{array}{l}\text { Low risk } \\
\text { All expected } \\
\text { outcomes } \\
\text { reported. }\end{array}$ & $\begin{array}{l}\text { Low risk } \\
\text { All } \\
\text { expected } \\
\text { outcomes } \\
\text { reported. }\end{array}$ & Low risk & Low risk \\
\hline $\begin{array}{l}\text { Rados } \\
\text { et al (67) }\end{array}$ & $\begin{array}{l}\text { Low risk } \\
\text { "Computer generated } \\
\text { randomization." }\end{array}$ & Unclear risk & $\begin{array}{l}\text { High risk } \\
\text { "The } \\
\text { participants } \\
\text { in the } \\
\text { study were } \\
\text { blinded } \\
\text { throughout the } \\
\text { study........ while } \\
\text { the authors were } \\
\text { not blinded." }\end{array}$ & $\begin{array}{l}\text { Low risk } \\
\text { All expected } \\
\text { outcomes } \\
\text { reported. }\end{array}$ & $\begin{array}{l}\text { Low risk } \\
\text { All } \\
\text { expected } \\
\text { outcomes } \\
\text { reported. }\end{array}$ & Low risk & Low risk \\
\hline $\begin{array}{l}\text { Ackerman } \\
\text { and Ahmad } \\
\text { (11) }\end{array}$ & $\begin{array}{l}\text { Low risk } \\
\text { "computer generated } \\
\text { randomization." }\end{array}$ & Unclear risk & High risk & $\begin{array}{l}\text { Low risk } \\
\text { All expected } \\
\text { outcomes } \\
\text { reported. }\end{array}$ & $\begin{array}{l}\text { Low } \\
\text { risk All } \\
\text { expected } \\
\text { outcomes } \\
\text { reported. }\end{array}$ & Low risk & Low risk \\
\hline $\begin{array}{l}\text { Kolsi et al } \\
\text { (69) }\end{array}$ & $\begin{array}{l}\text { High risk } \\
\text { "Immediately } \\
\text { before the injection } \\
\text { the patients were } \\
\text { randomized by the } \\
\text { physician who was to } \\
\text { perform the injection. } \\
\text { This physician did not } \\
\text { see the participants at } \\
\text { any other time during } \\
\text { the study." }\end{array}$ & High risk & Low risk & $\begin{array}{l}\text { Low risk } \\
\text { All expected } \\
\text { outcomes } \\
\text { reported. }\end{array}$ & $\begin{array}{l}\text { Low } \\
\text { risk All } \\
\text { expected } \\
\text { outcomes } \\
\text { reported. }\end{array}$ & Low risk & Low risk \\
\hline
\end{tabular}

Table 4. Newcastle-Ottowa Scale.

\begin{tabular}{|l|c|c|c|}
\hline & Selection & Comparability & Outcome \\
\hline Lee et al (73) & $\mathrm{XXXX}$ & $\mathrm{X}$ & $\mathrm{XX}$ \\
\hline Schaufele et al (70) & $\mathrm{XXXX}$ & $\mathrm{X}$ & $\mathrm{XX}$ \\
\hline Smith et al (72) & $\mathrm{XXXX}$ & $\mathrm{X}$ & $\mathrm{XX}$ \\
\hline
\end{tabular}

Table 5. United States Preventative Services Task Force Quality of Evidence Classification .

\begin{tabular}{|l|l|}
\hline I & At least 1 randomized controlled trial \\
\hline II-1 & Prospective, placebo-controlled trials that are not-randomized \\
\hline II-2 & Controlled retrospective studies \\
\hline II-3 & Uncontrolled retrospective studies \\
\hline III & Descriptive studies \\
\hline
\end{tabular}




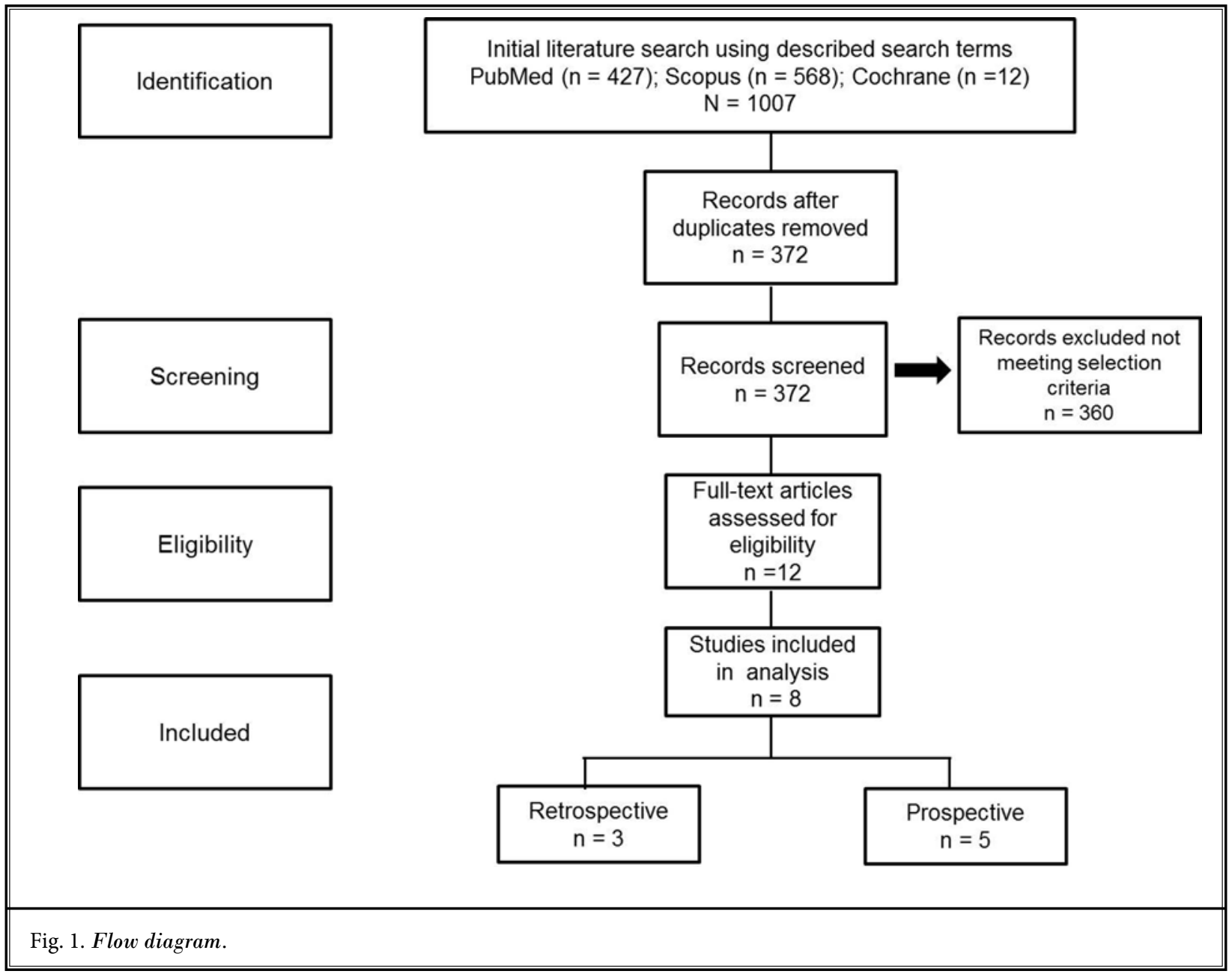

confirmation (61-63). Thus, 2 studies $(64,65)$ were excluded because of the lack of fluoroscopic guidance in performing injections. Direct comparative studies that did not meet inclusion/exclusion criteria are listed in the Table 6 . Thus 8 studies were considered for inclusion. Although some studies included caudal epidural steroid injection as part of their research protocol (11), only data on TFESI or ILESI were included for analysis.

\section{Screening for Methodological Quality of Studies}

Five out of 8 studies included were prospective and 3 were retrospective. The 5 prospective studies identified met the Cochrane Review criteria for randomized trials (41) (Table 2) and 3 studies met the Agency for Health Care Quality (AHCQ) criteria for observational studies (42). All studies surpassed 50 out of 100 on a modified and weighted Cochrane methodologic quality assessment criteria (66) (Table 1). A summary of study criteria for included prospective studies is listed in the Table 7. Retrospective studies were included in the review due to the paucity of prospective data (Table 2), but are not included in analysis of pain or functional improvement.

\section{Demographics Inclusion/Exclusion Criteria}

The total number of patients in all 8 studies was 506 , comprising 249 patients in the prospective studies, and 257 patients in the retrospective trials. In the 5 prospective trials, patients were followed up an average of 3.2 months, (range: 10 - 16 days following injection (11) for up to 6 months) $(37,64,67)$.

Statistical analysis was calculated utilizing the 5 prospective studies. There was a slight difference in 
Table 6. Direct comparative studies that did not meet inclusion/exclusion criteria.

\begin{tabular}{|l|l|l|l|l|l|l|}
\hline \multicolumn{1}{|c|}{ Author } & \multicolumn{1}{|c|}{ Study Type } & \multicolumn{1}{c|}{ Type of pain } & \multicolumn{1}{c|}{ TFESI } & \multicolumn{1}{c|}{ ILESI } & \multicolumn{1}{c|}{ Fluoroscopy } & Reason for Exclusion \\
\hline $\begin{array}{l}\text { Kraemer et al } \\
(60)\end{array}$ & $\begin{array}{l}\text { Prospective } \\
\text { randomized }\end{array}$ & Radicular pain & $\begin{array}{l}\text { Non- } \\
\text { conventional }\end{array}$ & Midline & Inconsistent use & Inconsistent fluoroscopy \\
\hline Lee et al (73) & $\begin{array}{l}\text { Prospective } \\
\text { randomized }\end{array}$ & $\begin{array}{l}\text { Axial back } \\
\text { pain without } \\
\text { radiation }\end{array}$ & Bilateral TFESI & $\begin{array}{l}\text { Midline or } \\
\text { posterior lateral }\end{array}$ & Yes & $\begin{array}{l}\text { Axial back pain not } \\
\text { radicular }\end{array}$ \\
\hline $\begin{array}{l}\text { Thomas et al } \\
(64)\end{array}$ & $\begin{array}{l}\text { Prospective } \\
\text { Randomized }\end{array}$ & Radicular pain & $\begin{array}{l}\text { Unilateral } \\
\text { TFESI } \\
\text { "safe triangle" }\end{array}$ & Midline & $\begin{array}{l}\text { ILESI performed } \\
\text { without contrast }\end{array}$ & $\begin{array}{l}\text { No contrast used for } \\
\text { fluoroscopy }\end{array}$ \\
\hline $\begin{array}{l}\text { Manchikanti et } \\
\text { al (65) }\end{array}$ & Retrospective & $\begin{array}{l}\text { Low back and } \\
\text { radicular pain }\end{array}$ & $\begin{array}{l}\text { Unilateral } \\
\text { TFESI }\end{array}$ & $\begin{array}{l}\text { Loss of } \\
\text { resistance }\end{array}$ & $\begin{array}{l}\text { ILESI without } \\
\text { fluoroscopy }\end{array}$ & No fluoroscopy \\
\hline
\end{tabular}

Table 7. Summary of Study criteria - prospective studies.

\begin{tabular}{|l|c|c|c|c||}
\hline \multicolumn{1}{|c|}{ Author } & Symptom & Cause of Pain & Baseline Pain Score & Duration of Symptoms \\
\hline Gharibo et al (68) & $\begin{array}{c}\text { unilateral lumbosacral } \\
\text { radicular pain }\end{array}$ & $\begin{array}{c}\text { Intervertebral herniated } \\
\text { disc }\end{array}$ & $\begin{array}{c}\text { ILESI: } 7.0 \pm 1.9 ; \\
\text { TFESI: } 6.4 \pm 2.1\end{array}$ & $>1$ Month $<1$ Year \\
\hline Candido et al (37) & $\begin{array}{c}\text { unilateral lumbosacral } \\
\text { radicular pain }\end{array}$ & $\begin{array}{c}\text { Intervertebral herniated } \\
\text { disc }\end{array}$ & $\begin{array}{c}\text { ILESI: } 6.78 \pm 2.44 ; \\
\text { TFESI: } 6.32 \pm 2.23\end{array}$ & $>15$ days \\
\hline Rados et al (67) & $\begin{array}{c}\text { unilateral lumbosacral } \\
\text { radicular pain }\end{array}$ & $\begin{array}{c}\text { Intervertebral herniated } \\
\text { disc excluding SS }\end{array}$ & $\begin{array}{c}\text { ILESI: } 7.36 \pm 1.6 ; \\
\text { TFESI: } 6.72 \pm 1.8\end{array}$ & $>6$ weeks $<1$ year \\
\hline $\begin{array}{l}\text { Ackerman and Ahmad } \\
(11)\end{array}$ & $\begin{array}{c}\text { unilateral lumbosacral } \\
\text { radicular pain }\end{array}$ & $\begin{array}{c}\text { Intervertebral herniated } \\
\text { disc }\end{array}$ & $\begin{array}{c}\text { ILESI: } 8.8 \pm 0.8 \\
\text { TFESI: } 8.6 \pm 0.9\end{array}$ & $\begin{array}{c}\text { ILESI } 33 \pm 7 \text { days, } \\
\text { TFESI } 35 \pm 5 \text { days }\end{array}$ \\
\hline Kolsi et al (69) & $\begin{array}{c}\text { unilateral lumbosacral } \\
\text { radicular pain }\end{array}$ & $\begin{array}{c}\text { Intervertebral herniated } \\
\text { disc }\end{array}$ & $\begin{array}{c}\text { ILESI: } 6.3 \pm 0.4 \\
\text { TFESI: } 7.0 \pm 0.4\end{array}$ & $>15$ days \\
\hline
\end{tabular}

gender distribution (62.5\% men received TFESI; $54.4 \%$ men received ILESI; $P<0.001$ ). The average age of patients receiving TFESI was $45.03 \pm 6.58$ years vs. $48.77 \pm$ 10.08 years in the ILESI group $(P<0.001)$. A majority of patients received injections at the L5-S1 level (64.3\%), vs. at the L4-L5 level (33.9\%) while only $1.8 \%$ were injected at either the L2-L3 or L3-L4 levels $(P=0.718)$.

\section{Type of Corticosteroids}

A majority of patients received either triamcinolone $(n=277 ; 55 \%$ ) or methylprednisolone acetate ( $n$ $=199 ; 39 \%)$. The remainder received cortivazol $(n=30$; $6 \%$ ), a synthetic agonist ligand that has a high affinity for the glucocorticoid receptor. There was an equal distribution of corticosteroids between TFESI and ILESI in all studies. The medication preparations for each study are summarized in Table 8.

\section{Procedure Techniques}

A variety of technical differences were noted between the studies in this review. Methodological differences in needle selection, use of fluoroscopy, and final needle tip position are summarized in Table 9.

\section{Outcomes and Clinical Significance}

Individual study outcomes and the presence of clinical significance are summarized below and in Table 8 .

\section{Pain Improvement as Efficacy}

All 5 prospective studies provided data regarding improvement in pain scores. Combined pain improvements looking at the end-time points in all 5 studies revealed a less than $20 \%$ difference between TFESI and ILESI (54.1\% vs. $42.7 \%$ ). Meta-analysis was performed, even though the heterogeneity was high ( $I^{2}$ up to $87 \%$ ), and showed slightly better pain improvement after TFESI injections only after 2 weeks of follow-up (Fig. 2b), with no difference noted after one and 6 month follow-ups (Fig. 2c, d).

Gharibo et al (68) followed patients for approximately 2 weeks (10 - 16 days). While Ackerman and Ahmad (11) followed patients for 24 weeks in their prospective study, and documented pain score improvements only after the first 2 weeks. Candido et al (37) also reported pain scores at 2 weeks and for up to six months. All 3 studies demonstrated an approximately $15 \%$ difference favoring efficacy from TFESI compared 
Table 8. Summary of head-to-head studies comparing TFESI vs. ILESI.

\begin{tabular}{|c|c|c|c|c|c|c|c|}
\hline Author & Study Type & \begin{tabular}{|l} 
Cause of \\
Lumbar \\
Radicular \\
Pain \\
\end{tabular} & TFESI & ILESI & $\begin{array}{l}\text { Duration } \\
\text { of Follow- } \\
\text { Up }\end{array}$ & \begin{tabular}{|l|} 
Pain \\
Improvement \\
TFESI vs. \\
ILESI \\
\end{tabular} & $\begin{array}{l}\text { Functional } \\
\text { Improvement } \\
\text { TFESI vs. } \\
\text { ILESI }\end{array}$ \\
\hline $\begin{array}{l}\text { Gharibo } \\
\text { et al (68) }\end{array}$ & \begin{tabular}{|l} 
Prospective \\
Randomized \\
Blinded \\
(Level 1)
\end{tabular} & $\begin{array}{l}\text { Lumbar Disc } \\
\text { Herniation } \\
\text { and/or } \\
\text { Spinal Stenosis }\end{array}$ & $\begin{array}{l}\mathrm{n}=20 \\
40 \mathrm{mg} \\
\text { triamcinolone } \\
+1 \mathrm{~mL} 0.25 \% \\
\text { bupivacaine } \\
\text { At } 2 \text { levels } \\
\text { Vol: } 4 \mathrm{~mL} \\
\end{array}$ & \begin{tabular}{|l}
$\mathrm{n}=18$ \\
$80 \mathrm{mg}$ \\
triamcinolone \\
$+2 \mathrm{~mL} 0.25 \%$ \\
bupivacaine \\
Vol: $4 \mathrm{~mL}$
\end{tabular} & 10-16 days & $73.4 \%$ vs. $44.3 \%$ & $43.6 \%$ vs. $49.3 \%$ \\
\hline $\begin{array}{l}\text { Candido } \\
\text { et al (37) }\end{array}$ & \begin{tabular}{|l|} 
Prospective \\
Randomized \\
Single-Blinded \\
(Level 1)
\end{tabular} & $\begin{array}{l}\text { Lumbar Disc } \\
\text { Herniation } \\
\text { and/or } \\
\text { Spinal Stenosis }\end{array}$ & \begin{tabular}{|l}
$\mathrm{n}=28$ \\
$80 \mathrm{mg}$ MPA \\
$+1 \mathrm{~mL} 1 \%$ \\
lidocaine +1 \\
mL NSS \\
Vol: $4 \mathrm{~mL}$ \\
\end{tabular} & $\begin{array}{l}\mathrm{n}=29 \\
80 \mathrm{mg} \text { MPA }+1 \\
\mathrm{~mL} 1 \% \text { lidocaine } \\
+1 \mathrm{~mL} \text { NSS } \\
\text { Vol: } 4 \mathrm{~mL}\end{array}$ & $\begin{array}{l}1 \text { month } \\
6 \text { months }\end{array}$ & $\begin{array}{l}16.5 \% \text { vs. } 23.1 \% \\
25.5 \% \text { vs. } 39.2 \%\end{array}$ & $\begin{array}{l}\text { N/A } \\
\text { N/A }\end{array}$ \\
\hline $\begin{array}{l}\text { Rados } \\
\text { et al (67) }\end{array}$ & $\begin{array}{l}\text { Prospective } \\
\text { Randomized } \\
\text { (Level 1) }\end{array}$ & $\begin{array}{l}\text { Lumbar Disc } \\
\text { Herniation } \\
\text { and/or } \\
\text { Spinal Stenosis }\end{array}$ & $\begin{array}{l}\mathrm{n}=32 \\
40 \mathrm{mg} \text { MPA } \\
+3 \mathrm{~mL} 0.5 \% \\
\text { lidocaine } \\
\text { Vol: } 5 \mathrm{~mL} \\
\end{array}$ & $\begin{array}{l}\mathrm{n}=32 \\
80 \mathrm{mg} \mathrm{MPA}+ \\
8 \mathrm{~mL} 0.5 \% \\
\text { lidocaine } \\
\text { Vol: } 10 \mathrm{~mL}\end{array}$ & 24 weeks & $45.6 \%$ vs. $43.5 \%$ & $28.3 \%$ vs. $25 \%$ \\
\hline $\begin{array}{l}\text { Ackerman } \\
\text { and Ahmad } \\
\text { (11) }\end{array}$ & $\begin{array}{l}\text { Prospective } \\
\text { Randomized } \\
\text { Blinded } \\
\text { (Level 1) }\end{array}$ & $\begin{array}{l}\text { Lumbar Disc } \\
\text { Herniation } \\
\text { and/or } \\
\text { Spinal Stenosis }\end{array}$ & $\begin{array}{l}\mathrm{n}=30 \\
40 \mathrm{mg} \\
\text { triamcinolone } \\
+4 \mathrm{~mL} \text { NSS } \\
\text { Vol: } 5 \mathrm{~mL}\end{array}$ & $\begin{array}{l}\mathrm{n}=30 \\
40 \mathrm{mg} \\
\text { triamcinolone }+ \\
4 \mathrm{~mL} \text { NSS } \\
\text { Vol: } 5 \mathrm{~mL}\end{array}$ & $\begin{array}{l}2 \text { weeks } \\
24 \text { weeks }\end{array}$ & $\begin{array}{l}72.1 \% \text { vs. } 35.2 \% \\
\text { N/A }\end{array}$ & $\begin{array}{l}53.3 \% \text { vs. } 60.6 \% \\
\text { N/A }\end{array}$ \\
\hline $\begin{array}{l}\text { Kolsi et al } \\
\text { (69) }\end{array}$ & \begin{tabular}{|l} 
Prospective \\
Randomized \\
Double- \\
Blinded
\end{tabular} & $\begin{array}{l}\text { Lumbar Disc } \\
\text { Herniation } \\
\text { and/or } \\
\text { Spinal Stenosis }\end{array}$ & $\begin{array}{l}\mathrm{n}=17 \\
3.75 \mathrm{mg} \\
\text { Cotivazol } \\
\text { Vol: } 1.5 \mathrm{~mL}\end{array}$ & $\begin{array}{l}\mathrm{n}=13 \\
3.75 \mathrm{mg} \\
\text { Cotivazol } \\
\text { Vol: } 1.5 \mathrm{~mL}\end{array}$ & 28 days & $62.8 \%$ vs. $63.5 \%$ & $34.8 \%$ vs. $50.9 \%$ \\
\hline $\begin{array}{l}\text { Smith et al } \\
(72)\end{array}$ & $\begin{array}{l}\text { Retrospective } \\
\text { Case-control } \\
\text { (Level II-3) }\end{array}$ & Spinal Stenosis & $\begin{array}{l}\mathrm{n}=19 \\
80 \mathrm{mg} \text { MPA } \\
+1-2 \mathrm{~mL} 2 \% \\
\text { lidocaine } \\
\text { Vol: } 3-4 \mathrm{~mL}\end{array}$ & $\begin{array}{l}\mathrm{n}=19 \\
80 \mathrm{mg} \mathrm{MPA}+ \\
2-3 \mathrm{~mL} 1 \% \\
\text { lidocaine } \\
\text { Vol: } 4-5 \mathrm{~mL}\end{array}$ & 4- 6 weeks & $30.5 \%$ vs. $39.5 \%$ & $\mathrm{~N} / \mathrm{A}$ \\
\hline Lee et al (73) & $\begin{array}{l}\text { Retrospective } \\
\text { Case-control } \\
\text { (Level 1) }\end{array}$ & $\begin{array}{l}\text { Lumbar Disc } \\
\text { Herniation } \\
\text { and/or } \\
\text { Spinal Stenosis }\end{array}$ & $\begin{array}{l}\mathrm{n}=115 \\
40 \mathrm{mg} \\
\text { triamcinolone } \\
+2 \text { or } 8 \mathrm{~mL} \\
0.5 \% \text { lidocaine } \\
\text { Vol: } 3 \text { or } 9 \mathrm{~mL}\end{array}$ & $\begin{array}{l}\mathrm{n}=64 \\
40 \mathrm{mg} \\
\text { triamcinolone }+8 \\
\mathrm{~mL} 0.5 \% \text { lidocaine } \\
\text { Vol: } 9 \mathrm{~mL}\end{array}$ & $\begin{array}{l}1 \text { month } \\
2 \text { months }\end{array}$ & $\begin{array}{l}78.0 \% \text { v. } 64.5 \% \\
68.2 \% \text { vs. } 51.6 \%\end{array}$ & $\begin{array}{l}\text { N/A } \\
\text { N/A }\end{array}$ \\
\hline $\begin{array}{l}\text { Schaufele } \\
\text { et al (70) }\end{array}$ & $\begin{array}{l}\text { Retrospective } \\
\text { Case-control } \\
\text { (Level 1) }\end{array}$ & $\begin{array}{l}\text { Lumbar Disc } \\
\text { Herniation } \\
\text { and/or } \\
\text { Spinal Stenosis }\end{array}$ & $\begin{array}{l}\mathrm{n}=20 \\
80 \mathrm{mg} \text { MPA } \\
+1-2 \mathrm{~mL} 2 \% \\
\text { lidocaine } \\
\text { Vol: } 3-4 \mathrm{~mL}\end{array}$ & $\begin{array}{l}\mathrm{n}=20 \\
80 \mathrm{mg} \mathrm{MPA}+ \\
2-3 \mathrm{~mL} 1 \% \\
\text { lidocaine } \\
\text { Vol: } 4-5 \mathrm{~mL}\end{array}$ & 2-3 weeks & $45.8 \%$ vs. $19.2 \%$ & N/A \\
\hline
\end{tabular}

Key: Vol = Total volume

with ILESI groups (54.7\% vs. $39.2 \%$ ). Gharibo et al (68) studied 38 patients, but did not provide long-term outcome follow-up (merely 10 - 16 days), and all injections were performed by a single practitioner. Patients from the TFESI group had statistically significantly better pain improvement, but all TFESI were performed using a 2-level, 2-needle technique (i.e., "double level TFESIs") with medication injected at adjacent contiguous neuroforamina.

Two prospective studies $(37,69))$ provided pain 
Table 9. Summary of techniques utilized.

\begin{tabular}{|c|c|c|c|c|c|c|}
\hline \multirow[b]{2}{*}{ Author } & \multicolumn{3}{|c|}{ TFESI } & \multicolumn{3}{|c|}{ ILESI } \\
\hline & Flouroscopy & Needle Type & $\begin{array}{c}\text { Needle } \\
\text { Position }\end{array}$ & Fluoroscopy & Needle Type & NeedlePosition \\
\hline $\begin{array}{l}\text { Gharibo et al } \\
(68)\end{array}$ & Contrast dye & $\begin{array}{l}\text { Two 22-gauge } \\
31 \frac{1}{2} \text {-inch } \\
\text { spinal needles }\end{array}$ & "safe triangle" & Contrast dye & 18-gauge Tuohy & $\begin{array}{l}\text { Interlaminar } \\
\text { Loss of resistance to air }\end{array}$ \\
\hline $\begin{array}{l}\text { Candido et al } \\
\text { (37) }\end{array}$ & Contrast dye & $\begin{array}{c}\text { 22-gauge } \\
31 \frac{1}{2} \text {-inchWhitacre }\end{array}$ & $\begin{array}{c}\text { Superior- } \\
\text { posteriorneuro- } \\
\text { foramen }\end{array}$ & Contrast dye & $\begin{array}{c}20 \text {-gauge } 3 \frac{1}{2} \text {-inch } \\
\text { Tuohy }\end{array}$ & $\begin{array}{l}\text { Parasagittal } \\
\text { Interlaminar Loss of } \\
\text { resistance to air }\end{array}$ \\
\hline $\begin{array}{l}\text { Rados et al } \\
(67)\end{array}$ & Contrast dye & 22-gauge needle & Not provided & Contrast dye & 19-gauge Touhy & $\begin{array}{l}\text { Interlaminar } \\
\text { Loss of resistance to air with } \\
\text { glass syringe }\end{array}$ \\
\hline $\begin{array}{l}\text { Ackerman } \\
\text { and Ahmad } \\
\text { (11) }\end{array}$ & Contrast dye & 22-gauge Touhy & $\begin{array}{l}\text { Superior- } \\
\text { posteriorneuro- } \\
\text { foramen }\end{array}$ & Contrast dye & 22-gauge Touhy & Not provided \\
\hline $\begin{array}{l}\text { Kolsi et al } \\
(69)\end{array}$ & Contrast dye & Not provided & $\begin{array}{c}\text { Nerve root } \\
\text { injection }\end{array}$ & Contrast dye & Not provided & Not provided \\
\hline $\begin{array}{l}\text { Smith et al } \\
(72)\end{array}$ & Contrast dye & $\begin{array}{l}\text { 25- or } 22 \text {-gauge } \\
31 \frac{1}{2} \text {-inch or } 5 \text {-inch } \\
\text { spinal needle }\end{array}$ & $\begin{array}{c}\text { Superior- } \\
\text { anteriorneuro- } \\
\text { foramen }\end{array}$ & Contrast dye & $\begin{array}{c}\text { 18-gauge } \\
31 / 2 \text {-inch or } 5 \text { inch } \\
\text { Tuohy }\end{array}$ & $\begin{array}{l}\text { Parasagittal } \\
\text { Interlaminar Loss of } \\
\text { resistance to air }\end{array}$ \\
\hline Lee et al (73) & Contrast dye & $\begin{array}{l}\text { 22-gauge spinal } \\
\text { needle }\end{array}$ & $\begin{array}{c}\text { Superior- } \\
\text { anteriorneuro- } \\
\text { foramen }\end{array}$ & Contrast dye & $\begin{array}{c}\text { 18-gauge } \\
31 \frac{12-\text {-inchTuohy }}{}\end{array}$ & $\begin{array}{l}\text { Interlaminar } \\
\text { Loss of resistance to air }\end{array}$ \\
\hline $\begin{array}{l}\text { Schaufele et al } \\
(70)\end{array}$ & Contrast dye & $\begin{array}{l}25 \text { - or } 22 \text {-gauge } \\
31 \frac{1}{2} \text {-inch or } 5 \text {-inch } \\
\text { spinal needle }\end{array}$ & $\begin{array}{l}\text { Superior- } \\
\text { anteriorneuro- } \\
\text { foramen }\end{array}$ & Contrast dye & $\begin{array}{c}\text { 18-gauge, } \\
\text { 31/2-inch or } 5 \text {-inch } \\
\text { Tuohy }\end{array}$ & $\begin{array}{l}\text { Parasagittal } \\
\text { Interlaminar Loss of } \\
\text { resistance to air }\end{array}$ \\
\hline
\end{tabular}

score data at one month following injections. Both studies demonstrated virtually no efficacy differences between approaches (34.0\% TFESI vs. $35.6 \%$ ILESI). Based on Cochrane review guidelines, this difference was not considered clinically significant. Kolsi et al (69) found no significant efficacy differences between TFESI and ILESI in the short- or long-term follow-ups. A limitation of this study was the small sample size $(n=$ 40). Two prospective studies $(37,67)$ followed patients for at least 6 months and provided data regarding pain improvement expressed as a numeric rating pain score. Both studies showed slightly better pain improvement after ILESI than TFESI injections ( $41.5 \%$ vs. $36.2 \%$ pain improvement). Candido et al (37) showed no difference in pain scores between TFESI and ILESI performed using a lateral parasagittal interlaminar approach, on short- (one month) or long-term (6 months) follow-ups. Rados et al (67) also showed no statistically significant difference between the 2 approaches. A major limitation of this study was the use of different protocols for injectate mixtures and volumes. Ackerman and Ahmad (11) showed better pain improvement at 24 weeks in a TFESI group. However, the pain improvement was graded as complete, partial, or no relief, and was not assessed using conventional numeric rating scale (NRS) scores. NRS scores were not used in the combined analysis for the 6-month follow up. They showed that out of 30 patients who had ILESI injections, $3(10 \%)$ reported complete pain relief, $15(50 \%)$ partial pain relief, and $12(40 \%)$ demonstrated no relief. Of the 30 patients who had TFESI injections, $9(30 \%)$ reported complete pain relief, $16(53 \%)$ had partial pain relief, and $5(17 \%)$ had no relief. However, different volumes and doses of medication were used in both approaches.

\section{Functional Improvement}

Four of 5 prospective studies included in our systematic review measured functional improvement as one of their study outcomes. Three studies $(11,67,68)$ used the Oswestry Disability Index (ODI and one used the EIFEL score (69), which is the French version of the RolandMorris Disability Questionnaire for low back pain. Metaanalysis was performed even though the heterogeneity was high $\left(I^{2}=65.5 \%\right)$, showing better functional improvement after ILESI injections (Fig. 3a, b).

Two studies provided data for functional improvement at approximately 2 weeks $(11,68)$ and showed a slightly better functional improvement in the ILESI 
Transforaminal Vs. Interlaminar Epidural Steroid Injections

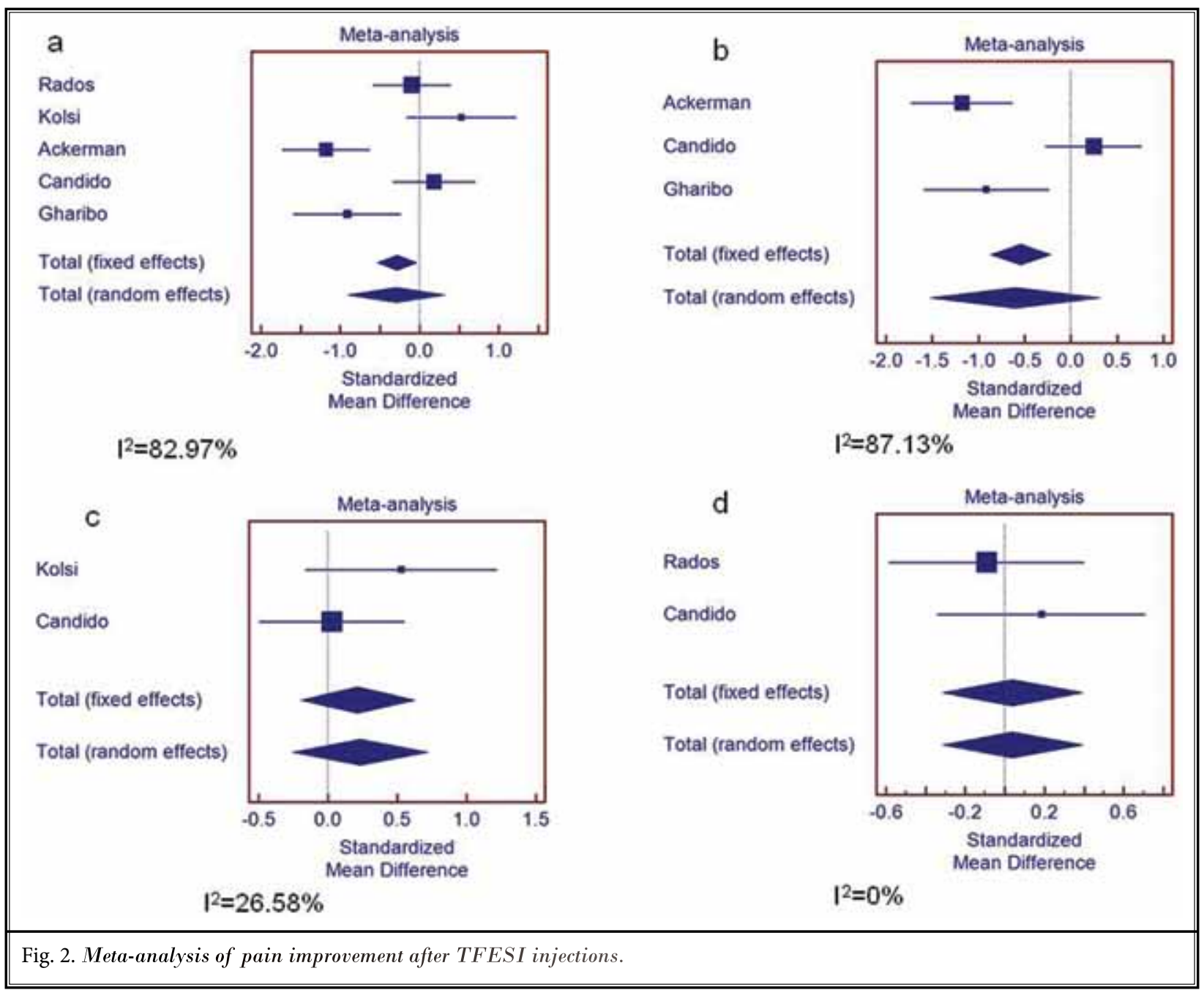

group (56.4\%) compared with the TFESI group (49.4\%). One study (69) provided data for a one-month followup and found significantly better functional improvement in the ILESI than in TFESI group (50.9\% vs. $34.8 \%)$. Also, one study (67) that followed patients for 6 months showed slightly better functional improvement in the TFESI group (28.3\%) than in the ILESI group (25.0\%). Combined data from all 4 studies showed only slight differences between these 2 approaches (TFESI $40.1 \%$ and ILESI $44.8 \%$ ).

\section{Other Outcomes}

Candido et al (37) used the contrast spread pattern between TFESI and ILESI approaches as the primary outcome measure in their study, along with total fluoroscopy time and pain relief as secondary measures. An independent blinded radiologist graded the lateral projection fluoroscopic images from each patient. Patients in the parasagittal ILESI group demonstrated more consistent anterior epidural spread (29 of 29; $100 \%)$, compared to 21 of $28(75 \%)$ patients in the TFESI group. Mean continuous fluoroscopy time was 28.96 seconds ( $95 \% \mathrm{Cl}, 23.9$ - 34.1 seconds) in the parasagittal ILESI group and 46.25 seconds $(95 \% \mathrm{Cl}, 36.27$ - 56.23 seconds) in the TF group ( $P=0.003$ ) (37). Ackerman and Ahmad (11) also looked at the contrast spread and pain relief at 24 weeks, finding that patients wherein ventral epidural spread was documented had more complete pain relief, while patients with non-ventral spread had more incomplete pain relief regardless of which approach was used.

\section{Level of Evidence}

In the treatment of lumbosacral radicular pain due 


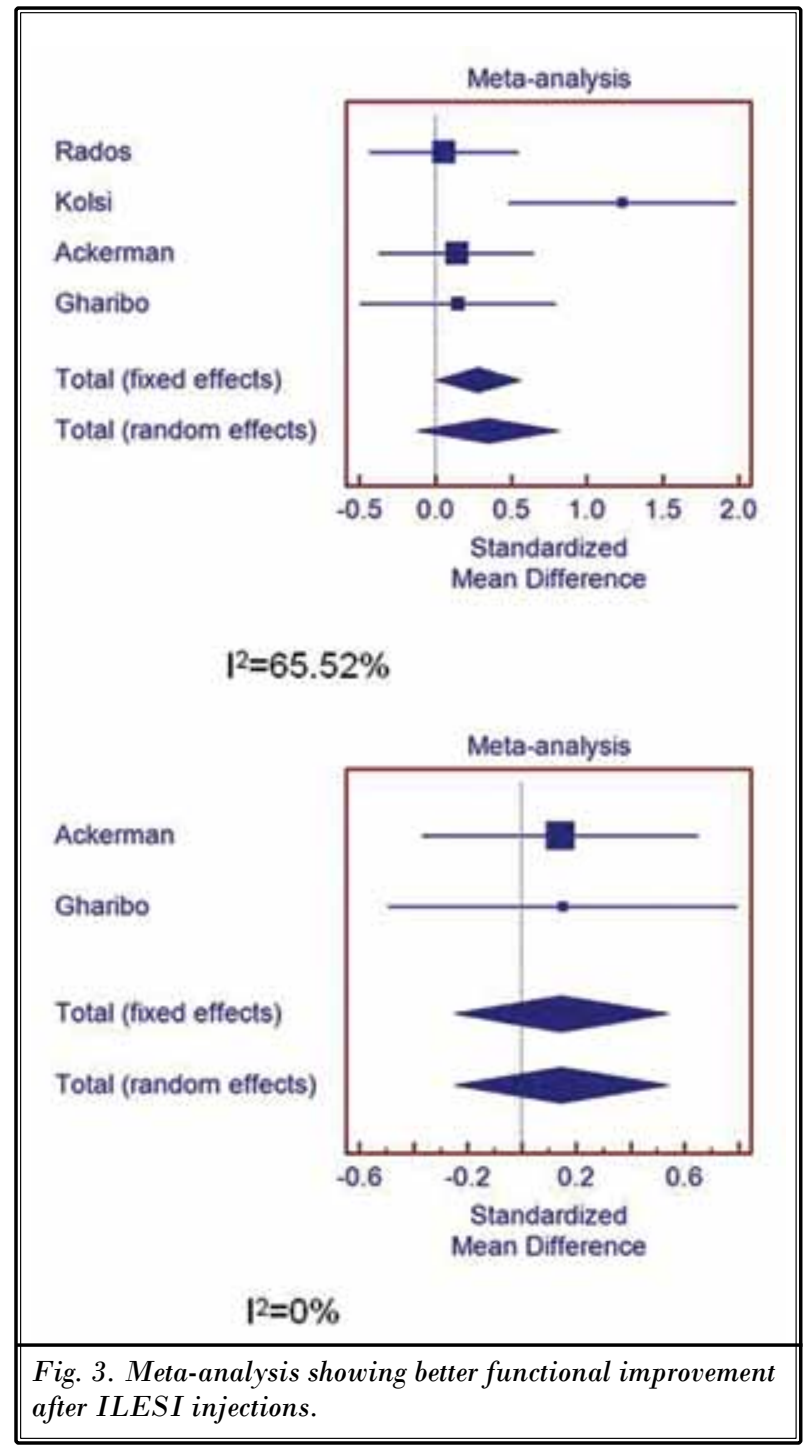

to disc herniation, there is Level 1 evidence for significant improvement in pain scores with fluoroscopically guided TFESI compared to fluoroscopically guided ILESI in both the short-term: 2 RCTs $(11,68)$, one retrospective study (RS) (70), and one long-term RCT (11). These findings are opposed by Level 1 evidence finding no difference between these groups in the short-term: 3 RCTs $(37,67,69)$, and long-term: 3 RCTs from the same authors $(31,67,69)$.

There is Level 1 evidence for no difference in improvement of function between fluoroscopically guided TFESI compared to fluoroscopically guided ILESI groups in both short-term: 3 RCTs $(11,67,68)$, and longterm: 2 RCTs $(11,67)$.

\section{Discussion}

The total number of patients identified in the 5 prospective studies that met criteria for this review was 249 . There was a $15 \%$ difference favoring efficacy of TFESI compared with ILESI only in the short-term (2 weeks) follow-up period. Studies that followed patients from between one to 6 months, as well as combined pain improvements when looking at end points "time," identified no clinical or statistically significant differences in efficacy between TFESI and ILESI. Four prospective studies included in our systematic review that measured functional improvement and that followed patients from between 2 weeks to one month found better functional improvement in the ILESI groups. However, none of these differences were considered clinically significant, according to Cochrane review guidelines (40).

For the treatment of unilateral lumbosacral radicular pain due to intervertebral disc herniation, the head-to-head evidence is mixed as to whether TFESI is superior to ILESI for clinically significant improvements in pain and functional outcomes, versus whether the 2 techniques are equivalent. We could find no study demonstrating superior efficacy outcomes of ILESI compared to TFESI for either pain reduction or functional improvement.

Although not shown in any of the studies within this review, TFESI have been shown to provide pain relief lasting upwards of 12 months (71). It is a widely held belief that increased deposition of medication into the ventral epidural space will result in greater efficacy and subsequent reduction in pain scores. We found only one head-to-head study to support this conclusion (11). Although Ackerman and Ahmad (11) performed a randomized, blinded prospective trial, the study results have limited clinical utility due to multiple methodological limitations including: the specific inclusion of radicular pain in an S1 dermatomal distribution; the use of repeat injections 2 weeks apart, as part of a series of 3 injections; and documented pain score improvement only after the first 2 weeks, even though patients were followed for 24 weeks (11). The majority of studies included in our review used a midline approach to ILESI. Currently, only one prospective study (37) and 2 retrospective studies $(70,72)$ have directly compared parasagittal (non-midline) ILESI to TFESI. Two studies found no difference for both short- and long-term pain outcomes between TFESI and parasagittal ILESI. Schaufele et al 
(70) found no difference in pain scores post-injection between TFESI and ILESI. However, the authors claimed a statistically significant superiority of TFESI for pain relief during a follow-up at up to 12 months. This conclusion is limited by repeated and uncontrolled use of additional epidural steroid injections and surgical interventions at undefined intervals during that 12 month period. There is evidence that a parasagittal ILESI approach may provide comparable ventral flow of corticosteroids (37) as well as similar pain relief and functional changes when compared to TFESI. Indeed, although midline ILESI epidurography patterns may demonstrate ventral epidural spread of the contrast as low as $36 \%$ of the time (1), one study in our review found ventral spread with parasagittal ILESI in $100 \%$ of subjects (37). As highlighted in the results section, this study demonstrated no difference in pain or functional outcomes between TFESI and parasagittal ILESI at 2 weeks, one, 3, and 6 months (37). Given the rare but serious complications associated with TFESI, further head-to-head study of parasagittal ILESI compared to TFESI appears to be warranted.

Some practitioners have attempted to utilize the purported advantages of the TF approach, i.e. deposition of the medication into the ventral epidural space, by accessing the neuroforamen via the interlaminar window (as an "inside-out" type of approach) $(73,74)$. These approaches are much less commonly performed than classical TFESI ("outside-in") and statistics for efficacy and complications are not available. There is no evidence that this type of approach decreases risks of morbidity or mortality associated with TFESI and further studies are necessary to delineate its role in the management of lumbosacral radicular pain.

The findings reported herein must take into account several important considerations and limitations: First, there is no consistency between the studies cited for inclusion and exclusion criteria. Secondly, there is no consistency or standardization of doses, injectate volumes or types of glucocorticoids utilized for either TFESI or ILESI between studies, or in the case of some studies (11) even between the different approaches used in the same study. There is no standardization of follow-up periods or for number or type of interval treatments, including additional injections, performed in either group. No consensus was identified between the need for addition or lack of addition of local anesthetic to the steroid, or to the type of local anesthetic or dose used, which was disparate in all studies. A standardized approach to the interlaminar space for ILESI and to the intervertebral foramen during TFESI was notably absent. Needle type, gauge, and rate of injection also varied among studies. Statistical methodologies were also applied in a disparate manner in many of the studies which met inclusion criteria.

\section{Conclusion}

Based on the 5 randomized, controlled trials consisting of 249 subjects which directly compared TFESI to ILESI for unilateral lumbosacral pain secondary to disc herniation/degeneration, there is high quality evidence to support a finding of no clinically significant difference in efficacy for pain relief or functional improvement between the 2 techniques at all follow-up intervals. This limited sample of studies has potentially profound implications for the clinical practice of interventional pain medicine. Current practice trends have demonstrated a shift away from interlaminar epidural steroid injections, towards the increasingly more widespread practice of the transforaminal approach (17), in part due to the unsubstantiated belief of superior efficacy. This perceived superiority of TFESI is accompanied by potential additional risks, likely to be much less common with ILESI, such as intra-discal and intravascular injection with the attendant sequelae. Additionally, though TFESI and ILESI have been shown in prospective trials to be efficacious for pain relief greater than 6 months (71), there is insufficient direct comparative literature addressing differences in outcomes between the 2 techniques beyond 6 -months of follow-up. This begs the question as to whether the increased risk of potential catastrophic morbidity is effectively offset by the minimal differences in efficacy between the 2 respective approaches. While some of the increased risks associated with TFESI may be ameliorated by removal of particulate corticosteroid from the injectate, numerous studies have demonstrated that corticosteroid use itself may have marginal benefit in epidural injections (75-78).

If one elects to choose a neuraxial steroid injection as part of a multi-modal approach to the management of unilateral radicular pain, the risks versus benefits of each approach must be taken into consideration. Although each technique poses its own unique characteristic set of risks, it appears that their efficacy in terms of pain relief or improvement in functioning is not significantly different. Future studies are necessary to confirm the findings of this systematic review, including 
larger numbers of subjects and with standardization of approaches, doses, and inclusion/exclusion criteria may help resolve any ongoing controversies involving a comparison of selecting either interlaminar vs. transforaminal administration of corticosteroids for unilateral radicular type pain.

\section{Conflict of Interest}

Each author certifies that he or she, or a member of his or her immediate family, has no commercial association (i.e., consultancies, stock ownership, equity interest, patent/licensing arrangements, etc.) that might pose a conflict of interest in connection with the submitted manuscript.

\section{References}

1. Botwin KP, Natalicchio J, Hanna A. Fluoroscopic guided lumbar interlaminar epidural injections: A prospective evaluation of epidurography contrast patterns and anatomical review of the epidural space. Pain Physician 2004; 7:77-80.

2. Kawakami M, Weinstein JN, Chatani K, Spratt KF, Meller ST, Gebhart GF. Experimental lumbar radiculopathy. Behavioral and histologic changes in a model of radicular pain after spinal nerve root irritation with chromic gut ligatures in the rat. Spine (Phila Pa 1976) 1994; 19:1795-1802.

3. Kawakami M, Weinstein JN, Spratt KF, Chatani K, Traub RJ, Meller ST, Gebhart GF. Experimental lumbar radiculopathy. Immunohistochemical and quantitative demonstrations of pain induced by lumbar nerve root irritation of the rat. Spine (Phila Pa 1976) 1994; 19:1780-1794.

4. Katz WA, Rothenberg R. Section 3: The nature of pain: Pathophysiology. J Clin Rheumatol 2005; 11:S11-S15.

5. Lee HM, Weinstein JN, Meller ST, Hayashi N, Spratt KF, Gebhart GF. The role of steroids and their effects on phospholipase A2. An animal model of radiculopathy. Spine (Phila Pa 1976) 1998; 23:1191-1196.

6. Harrast MA. Epidural steroid injections for lumbar spinal stenosis. Curr Rev Musculoskelet Med 2008; 1:32-38.

7. Cuellar JM, Golish SR, Reuter MW, Cuellar VG, Angst MS, Carragee EJ, Yeomans DC, Scuderi GJ. Cytokine evaluation in individuals with low back pain using discographic lavage. Spine J 2010; 10:212-218

8. Scuderi GJ, Cuellar JM, Cuellar VG, Yeomans DC, Carragee EJ, Angst MS. Epidural interferon gamma-immunoreactivity: A biomarker for lumbar nerve root irritation. Spine (Phila Pa 1976) 2009; 34:2311-2317.
9. Tachihara H, Sekiguchi M, Kikuchi S, Konno S. Do corticosteroids produce additional benefit in nerve root infiltration for lumbar disc herniation? Spine (Phila Pa 1976) 2008; 33:743-747.

10. Lutz GE, Vad VB, Wisneski RJ. Fluoroscopic transforaminal lumbar epidural steroids: An outcome study. Arch Phys Med Rehabil 1998; 79:1362-1366.

11. Ackerman WE, 3rd, Ahmad M. The efficacy of lumbar epidural steroid injections in patients with lumbar disc herniations. Anesth Analg 2007; 104:1217-1222.

12. Buenaventura RM, Datta S, Abdi S, Smith HS. Systematic review of therapeutic lumbar transforaminal epidural steroid injections. Pain Physician 2009; 12:233-251.

13. Carette $S$, Leclaire R, Marcoux S, Morin F, Blaise GA, St-Pierre A, Truchon R, Parent $F$, Levesque J, Bergeron V, Montminy $P$, Blanchette C. Epidural corticosteroid injections for sciatica due to herniated nucleus pulposus. N Engl J Med 1997; 336:1634-1640.

14. Parr AT, Diwan S, Abdi S. Lumbar interlaminar epidural injections in managing chronic low back and lower extremity pain: A systematic review. Pain Physician 2009; 12:163-188.

15. Vad VB Bhat AL, Lutz GE, Cammisa F. Transforaminal epidural steroid injections in lumbosacral radiculopathy: A prospective randomized study. Spine (Phila $\mathrm{Pa}$ 1976) 2002; 27:11-16. \{There are two number 15 references.

16. Manchikanti L, Pampati V, Falco FJE, Hirsch JA. Assessment of the growth of epidural injections in the Medicare population from 2000 to 2011. Pain Physician 2013; 16:E349-364

17. Manchikanti L, Pampati V, Falco FJE, Hirsch JA. Growth of spinal interventional pain management techniques: Analysis of utilization trends and Medicare ex- penditures 2000 to 2008. Spine (Phila Pa 1976) 2013; 38:157-168.

18. Nahm FS, Lee CJ, Lee SH, Kim TH, Sim WS, Cho HS, Park SY, Kim YC, Lee SC. Risk of intravascular injection in transforaminal epidural injections. Anaesthesia 2010; 65:917-921.

19. Chang Chien GC, Candido KD, Knezevic NN. Digital subtraction angiography does not reliably prevent paraplegia associated with lumbar transforaminal epidural steroid injection. Pain Physician 2012; 15:515-523.

20. Chung JY, Han JH, Kang JM, Lee BJ. Paraplegia after epidural steroid injection. Anaesth Intensive Care 2012; 40:1074-1076.

21. Kennedy DJ, Dreyfuss P, Aprill CN, Bogduk N. Paraplegia following imageguided transforaminal lumbar spine epidural steroid injection: Two case reports. Pain Med 2009; 10:1389-1394.

22. Candido KD, Katz JA, Chinthagada M, McCarthy RA, Knezevic NN. Incidence of intradiscal injection during lumbar fluoroscopically guided transforaminal and interlaminar epidural steroid injections. Anesth Analg 2010; 110:1464-1467.

23. Cohen SP, Maine DN, Shockey SM, Kudchadkar S, Griffith S. Inadvertent disk injection during transforaminal epidural steroid injection: Steps for prevention and management. Pain Med 2008; 9:688-694.

24. Hooten WM, Mizerak A, Carns PE, Huntoon MA. Discitis after lumbar epidural corticosteroid injection: A case report and analysis of the case report literature. Pain Med 2006; 7:46-51.

25. Goodman BS, Bayazitoglu M, Mallempati S, Noble BR, Geffen JF. Dural puncture and subdural injection: $A$ complication of lumbar transforaminal epidural injections. Pain Physician 2007; 10:697-705. 
26. Siddiqui $M N$, Ranasinghe JS, Siddiqui S. Epidural hematoma after epidural steroid injection: A possible association with use of pentosan polysulfate sodium. Anesthesiology 2001; 95:1307.

27. Horlocker TT, Bajwa ZH, Ashraf Z, Khan $\mathrm{S}$, Wilson JL, Sami N, Peeters-Asdourian C, Powers CA, Schroeder DR, Decker PA, Warfield CA. Risk assessment of hemorrhagic complications associated with nonsteroidal antiinflammatory medications in ambulatory pain clinic patients undergoing epidural steroid injection. Anesth Analg 2002; 95:1691-1697.

28. Ain RJ, Vance MB. Epidural hematoma after epidural steroid injection in a patient withholding enoxaparin per guidelines. Anesthesiology 2005; 102:701-703.

29. Ozdemir O, Calisaneller T, Yildirim E, Altinors N. Acute intracranial subdural hematoma after epidural steroid injection: A case report. J Manipulative Physiol Ther 2007; 30:536-538.

30. Yoo HS, Park SW, Han JH, Chung JY, Yi JW, Kang JM, Lee BJ, Kim DO. Paraplegia caused by an epidural hematoma in a patient with unrecognized chronic idiopathic thrombocytopenic purpura following an epidural steroid injection. Spine (Phila Pa 1976) 2009; 34:E376-379.

31. Bilir A, Gulec $S$. Cauda equina syndrome after epidural steroid injection: A case report. J Manipulative Physiol Ther 2006; 29:492 e1-3.

32. Riew KD, Yin $Y$, Gilula L, Bridwell $K H$, Lenke LG, Lauryssen C, Goette K. The effect of nerve-root injections on the need for operative treatment of lumbar radicular pain. A prospective, randomized, controlled, double-blind study. J Bone Joint Surg Am 2000; 82-A:1589-1593.

33. Fantini GA, Pawar AY. Access related complications during anterior exposure of the lumbar spine. World J Orthop 2013; 4:19-23.

34. Inamasu J, Guiot BH. Vascular injury and complication in neurosurgical spine surgery. Acta Neurochir (Wien) 2006; 148:375-387.

35. Wood KB, Devine J, Fischer D, Dettori $J R$, Janssen $M$. Vascular injury in elective anterior lumbosacral surgery. Spine (Phila Pa 1976) 2010; 35:S66-S75.

36. Manchikanti L, Boswell MV, Singh V, Benyamin RM, Fellows B, Abdi S, Buenaventura RM, Conn A, Datta S, Derby R, Falco FJ, Erhart S, Diwan S, Hayek SM, Helm S, Parr AT, Schultz DM, Smith HS, Wolfer LR, Hirsch JA. Comprehensive evidence-based guidelines for interventional techniques in the manage- ment of chronic spinal pain. Pain Physician 2009; 12:699-802.

37. Candido KD, Raghavendra MS, Chinthagada M, Badiee S, Trepashko DW. A prospective evaluation of iodinated contrast flow patterns with fluoroscopically guided lumbar epidural steroid injections: The lateral parasagittal interlaminar epidural approach versus the transforaminal epidural approach. Anesth Analg 2008; 106:638-644.

38. Lee JH, An JH, Lee SH. Comparison of the effectiveness of interlaminar and bilateral transforaminal epidural steroid injections in treatment of patients with lumbosacral disc herniation and spinal stenosis. Clin J Pain 2009; 25:206-210.

39. Manchikanti L, Benyamin RM, Helm $\mathrm{S}$, Hirsch JA. Evidence-based medicine, systematic reviews, and guidelines in interventional pain management: Part 3: Systematic reviews and meta-analyses of randomized trials. Pain Physician 2009; 12:35-72.

40. Furlan AD, Pennick V, Bombardier C, van Tulder M; Editorial Board, Cochrane Back Review Group. 2009 updated method guidelines for systematic reviews in the Cochrane Back Review Group. Spine (Phila Pa 1976) 2009; 34:1929-1941.

41. Koes BW, Scholten RJ, Mens JM, Bouter LM. Efficacy of epidural steroid injections for low-back pain and sciatica: a systematic review of randomized clinical trials. Pain 1995; 63:279-288.

42. West S, King V, Carey TS, Lohr KN, McKoy N, Sutton SF, Lux L. Systems to rate the strength of scientific evidence. Evid Rep Technol Assess (Summ) 2002; nneed issue\} 1-11.

43. Nelemans PJ, deBie RA, deVet HC, Sturmans $F$. Injection therapy for subacute and chronic benign low back pain. Spine (Phila Pa 1976) 2001; 26:501-515.

44. Conn A, Buenaventura RM, Datta $S$, Abdi S, Diwan S. Systematic review of caudal epidural injections in the management of chronic low back pain. Pain Physician 2009; 12:109-135.

45. Helm S, Hayek SM, Benyamin RM, Manchikanti L. Systematic review of the effectiveness of thermal annular procedures in treating discogenic low back pain. Pain Physician 2009; 12:207-232

46. Patel VB, Manchikanti L, Singh V, Schultz DM, Hayek SM, Smith HS. Systematic review of intrathecal infusion systems for long-term management of chronic non-cancer pain. Pain Physician 2009; 12:345-360.
47. Frey ME, Manchikanti L, Benyamin RM, Schultz DM, Smith HS, Cohen SP. Spinal cord stimulation for patients with failed back surgery syndrome: a systematic review. Pain Physician 2009; 12:379-397.

48. Benyamin RM, Singh V, Parr AT, Conn A, Diwan S, Abdi S. Systematic review of the effectiveness of cervical epidurals in the management of chronic neck pain. Pain Physician 2009; 12:137-157.

49. Epter RS, Helm S, 2nd, Hayek SM, Benyamin RM, Smith HS, Abdi S. Systematic review of percutaneous adhesiolysis and management of chronic low back pain in post lumbar surgery syndrome. Pain Physician 2009; 12:361-378.

50. Hayek SM, Helm S, Benyamin RM, Singh V, Bryce DA, Smith HS. Effectiveness of spinal endoscopic adhesiolysis in post lumbar surgery syndrome: $A$ systematic review. Pain Physician 2009; 12:419-435.

51. Falco FJ, Erhart S, Wargo BW, Bryce DA, Atluri S, Datta S, Hayek SM. Systematic review of diagnostic utility and therapeutic effectiveness of cervical facet joint interventions. Pain Physician 2009; 12:323-344.

52. Datta S, Lee M, Falco FJ, Bryce DA, Hayek SM. Systematic assessment of diagnostic accuracy and therapeutic utility of lumbar facet joint interventions. Pain Physician 2009; 12:437-460.

53. Atluri S, Datta S, Falco FJ, Lee M. Systematic review of diagnostic utility and therapeutic effectiveness of thoracic facet joint interventions. Pain Physician 2008; 11:611-629.

54. Manchikanti L, Dunbar EE, Wargo BW, Shah RV, Derby R, Cohen SP. Systematic review of cervical discography as a diagnostic test for chronic spinal pain. Pain Physician 2009; 12:305-321.

55. Wolfer LR, Derby R, Lee JE, Lee SH. Systematic review of lumbar provocation discography in asymptomatic subjects with a meta-analysis of false-positive rates. Pain Physician 2008; 11:513-538.

56. Singh V, Manchikanti L, Shah RV, Dunbar EE, Glaser SE. Systematic review of thoracic discography as a diagnostic test for chronic spinal pain. Pain Physician 2008; 11:631-642.

57. Smith HS, Chopra P, Patel VB, Frey ME, Rastogi R. Systematic review of the role of sedation in diagnostic spinal interventional techniques. Pain Physician 2009; 12:195-206.

58. Rupert MP, Lee M, Manchikanti L, Datta S, Cohen SP. Evaluation of sacroiliac 
joint interventions: A systematic appraisal of the literature. Pain Physician 2009; 12:399-418.

59. Berg AO, Allan JD. Introducing the third US Preventive Services Task Force. Am J Prev Med 2001; 20:3-4.

6o. Kraemer J, Ludwig J, Bickert U, Owczarek V, Traupe M. Lumbar epidural perineural injection: A new technique. Eur Spine J 1997; 6:357-361.

61. Liu SS, Melmed AP, Klos JW, Innis CA. Prospective experience with a 20-gauge Tuohy needle for lumbar epidural steroid injections: Is confirmation with fluoroscopy necessary? Reg Anesth Pain Med 2001; 26:143-146.

62. Manchikanti L, Bakhit CE, Pakanati $R R$, Fellows B. Fluoroscopy is medically necessary for the performance of epidural steroids. Anesth Analg 1999; 89:1330-1331.

63. Johnson BA, Schellhas KP, Pollei SR. Epidurography and therapeutic epidural injections: technical considerations and experience with 5334 cases. AJNR Am J Neuroradiol 1999; 20:697-705.

64. Thomas E, Cyteval C, Abiad L, Picot MC Taourel P, Blotman F. Efficacy of transforaminal versus interspinous corticosteroid injection in discal -radiculgia - a prospective, randomised, double-blind study. Clin Rheumatol 2003; 22:299-304.

65. Manchikanti L, Pakanati R, Pampati V. Comparison of three routes of epidural steroid injections in low back pain. Pain Digest 1999; 9:277-285.

66. Manchikanti L, Singh V, Derby $R$, Schultz DM, Benyamin RM, Prager JP, Hirsch JA. Reassessment of evidence synthesis of occupational medicine practice guidelines for interventional pain management. Pain Physician 2008; 11:393-482.

67. Rados I, Sakic K, Fingler M, Kapural L. Efficacy of interlaminar vs transforaminal epidural steroid injection for the treatment of chronic unilateral radicular pain: Prospective, randomized study. Pain Med 2011; 12:1316-1321.

68. Gharibo CG, Varlotta GP, Rhame EE, Liu EC, Bendo JA, Perloff MD. Interlaminar versus transforaminal epidural steroids for the treatment of subacute lumbar radicular pain: A randomized, blinded, prospective outcome study. Pain Physician 2011; 14:499-511.

69. Kolsi I, Delecrin J, Berthelot JM, Thomas L, Prost A, Maugars Y. Efficacy of nerve root versus interspinous injections of glucocorticoids in the treatment of diskrelated sciatica. A pilot, prospective, randomized, double-blind study. Joint Bone Spine 2000; 67:113-118.

70. Schaufele MK, Hatch L, Jones W. Interlaminar versus transforaminal epidural injections for the treatment of symptomatic lumbar intervertebral disc herniations. Pain Physician 2006; 9:361-366.

71. Ghahreman A, Ferch R, Bogduk N. The efficacy of transforaminal injection of steroids for the treatment of lumbar radicular pain. Pain Med 2010; 11:1149-1168.

72. Smith CC, Booker T, Schaufele MK, Weiss P. Interlaminar versus transforaminal epidural steroid injections for the treatment of symptomatic lumbar spinal stenosis. Pain Med 2010; 11:1511-1515.
73. Lee JH, Moon J, Lee SH. Comparison of effectiveness according to different approaches of epidural steroid injection in lumbosacral herniated disk and spinal stenosis.] Back Musculoskelet Rehabil 2009; 22:83-89.

74. Choi YK, Barbella JD. Evaluation of epidurographic contrast patterns with fluoroscopic-guided lumbar interlaminar ventral epidural injection. Pain Pract 2009; 9:275-281.

75. Manchikanti L, Singh V, Cash KA, Pampati V, Falco FJ. A randomized, doubleblind, active-control trial of the effectiveness of lumbar interlaminar epidural injections in disc herniation. Pain Physician 2014; 17:E61-E74.

76. Manchikanti L, Cash KA, McManus CD, Pampati V, Benyamin R. Fluoroscopic lumbar interlaminar epidural injections in managing chronic lumbar axial or discogenic pain. J Pain Res 2012; 5:301-311.

77. Benyamin RM, Manchikanti L, Parr AT, Diwan S, Singh V, Falco FJ, Datta S, Abdi $S$, Hirsch JA. The effectiveness of lumbar interlaminar epidural injections in managing chronic low back and lower extremity pain. Pain Physician 2012; 15:E363-404.

78. Manchikanti L, Cash KA, McManus CD, Pampati V, Fellows B. Fluoroscopic caudal epidural injections with or without steroids in managing pain of lumbar spinal stenosis: One-year results of randomized, double-blind, active-controlled trial. J Spinal Disord Tech 2012; 25:226-234 